\section{Knockdown of VEGFR2 inhibits proliferation and induces apoptosis in hemangioma- derived endothelial cells}

\author{
J.M. Ou, ${ }^{1}$ Z.Y. Yu, ${ }^{2}$ M.K. Qiu, ${ }^{1}$ Y.X. Dai, ${ }^{1}$ \\ Q. Dong, ${ }^{1}$ J. Shen, ${ }^{1}$ P. Dong, ${ }^{1}$ X.F. Wang, \\ Y.B. Liu, ${ }^{1}$ Z.W. Quan, ${ }^{1}$ Z.W. Fei ${ }^{3}$ \\ 'Department of General Surgery, Xinhua \\ Hospital affiliated to Shanghai Jiaotong \\ University School of Medicine, Shanghai \\ 2Department of General Surgery, Su Zhou \\ Municipal Hospital, Su Zhou \\ ${ }^{3}$ Department of General Surgery, Xinhua \\ Hospital (Chong Ming) affiliated \\ to Shanghai Jiaotong University School \\ of Medicine, Shanghai, China
}

\section{Abstract}

Angiogenesis is a process of development and growth of new capillary blood vessels from pre-existing vessels. Angiogenic growth factors play important roles in the development and maintenance of some malignancies, of which vascular endothelial growth factor (VEGF)/NEGFR2 interactions are involved in proliferation, migration, and survival of many cancer cells. The aim of this study was to investigate the function of VEGFR2 in human hemangiomas (HAs). Using immunohistochemistry assay, we examined the expression levels of VEGF, VEGFR2, Ki-67, glucose transporter-1 (Glut-1), phosphorylated protein kinase B (p-AKT) and p-ERK in different phases of human HAs. Positive expression of VEGF, VEGFR2, Ki-67, Glut-1, p-AKT and p-ERK was significantly increased in proliferating phase HAs, while decreased in involuting phase HAs $(\mathrm{P}=0.001 ; \mathrm{P}=0.003)$. In contrast, cell apoptotic indexes were decreased in proliferating phase HAs, but increased in involuting phase HAs $(\mathrm{P}<0.01)$. Furthermore, we used small hairpin RNA (shRNA)-mediated VEGFR2 knockdown in primary HA-derived endothelial cells (HemECs) to understand the role of VEGF/VEGFR2 signaling. Knockdown of VEGFR2 by Lv-shVEGFR2 inhibited cell viability and induced apoptosis in primary HemECs companied with decreased expression of $\mathrm{p}$ AKT, p-ERK, p-p38MAPK and Ki-67 and increased expression of caspase-3 (CAS-3); Overexpression of VEGFR2 promoted cell viability and blocked apoptosis in Lv-VEGFR2transfected HemECs. Taken together, our findings demonstrate that, increased expression of VEGFR2 is involved in the development of primary HemECs possibly through regulation of the AKT and ERK pathways, suggesting that
VEGFR2 may be a potential therapeutic target for HAs.

\section{Introduction}

Hemangiomas (HAs) are benign neoplasms of the vasculature, and these lesions, often referred to as infantile HAs, are considered to be the most common tumors of infancy. It is estimated that one in every ten children develops HAs, most of which are on the head or neck. ${ }^{1}$ Currently, one of the urgent tasks is to discover the molecular mechanisms involved in the development of HAs as well as the reliable biomarkers and possible therapeutic targets. $^{2}$

Vascular endothelial growth factors (VEGFs) belong to the platelet-derived growth factor supergene family, and play central roles in the regulation of angiogenesis. Its receptor vascular endothelial growth factor receptor-2 (VEGFR-2) exhibits a strong tyrosine kinase activity towards pro-angiogenic signals, and regulates endothelial cell proliferation, migration, vascular permeability, secretion and other endothelial functions. ${ }^{3}$ Some studies have shown that, VEGF and VEGFR2 are highly expressed in invasive breast cancer, and can predict the unfavorable survival of patients. ${ }^{4}$ Also, VEGFR-2 polymorphisms can be considered as the prognostic marker for tumor recurrence and overall survival in non-small-cell lung cancer (NSCLC). ${ }^{5}$ Moreover, activation of VEGFR2 by VEGF may be important signaling mechanisms involved in the progression and subsequent metastatic spread of prostate cancer. $^{6}$ VEGF protein can act on MMP-2, -7 and -9 via its receptor to strengthen cell invasion ability in colon cancer. ${ }^{7}$ Therefore, some therapeutic approaches using anti-VEGF/VEGFR2 can be made to inhibit proliferation and induce apoptosis of malignant cells in hematologic diseases. ${ }^{8}$

HAs are tumors formed by hyper-proliferation of vascular endothelial cells, which is caused by elevated VEGF signaling through VEGFR2. ${ }^{9}$ VEGFR2 gene (KDR) mutation is detected in Juvenile Has tissue but not in adjacent normal tissue, suggesting that one potential mechanism involved in HA formation is the alteration of the VEGF/NEGFR2 signaling pathway in endothelial cells. ${ }^{10}$ Some studies have found that VEGFR2 expression is increased in HA patients, and contribute to the formation of the vascular tumors. ${ }^{11}$ Interestingly, the proliferative phase of HAs is the result of constitutively high levels of VEGF-dependent signaling through VEGFR2,12 and VEGFR2 antibody can suppress the activity of VEGF through blocking the VEGFR2 signaling pathways including downstream AKT and ERK pathways, indicating
Correspondence: Prof. Zhe-Wei Fei, Department of General Surgery, Chongming Branch Hospital, Xinhua Hospital affiliated to Shanghai Jiaotong University School of Medicine, Shanghai Chongming County, No.25 Nan men gang Road, 202150 Shanghai, China.

Tel. $+86.21 .25078999-7885$ - Fax: +86.21 .65675173 . E-mail: zheweifei@163.com.

Key words: vascular endothelial growth factor receptor 2, hemangioma, proliferation, apoptosis.

Contributions: JMO, ZYY, contribute equally to this work; ZF, experimental arrangement and design; JMO, experiment performing and manuscript writing: ZYY, experiment performing; $\mathrm{YBL}$ experimental arrangement; ZWQ, data analysis, other authors, data collection.

Conflict of interests: the authors declare no conflict of interests.

Funding: this work was supported by Shangha Science and Technology Committee Scientific and Technological Innovation Project (No. 12140901102) and Shanghai City Board of Education Research and Innovation Project (No. 12YZ042).

Received for publication: 17 August 2013. Accepted for publication: 3 February 2014.

This work is licensed under a Creative Commons Attribution NonCommercial 3.0 License (CC BYNC 3.0).

(O)Copyright J.M. Ou et al., 2014

Licensee PAGEPress, Italy

European Journal of Histochemistry 2014; 58:2263 doi:10.4081/ejh.2014.2263

the potential applications of anti-VEGFR2 antibody in the treatment of Has. ${ }^{13}$ However, some studies give the opposite view that the levels of serum soluble VEGFR2 are slightly lower in proliferating phase HA patients compared to involuting phase ones, indicating the possible dysregulation of VEGFR2 receptor, ${ }^{14}$ while VEGF inhibits tumor cell invasion and mesenchymal transition through a MET/NEGFR2 complex in glioblastoma multiforme. ${ }^{15}$ Thus, it is indispensable to further explore the function of VEGF/NEGFR2 signaling in cancer. In the present study, we studied the expression levels of VEGF and VEGFR2 in different phases of human HA. Through shRNA-mediated VEGFR2 knockdown in primary HemECs, we observed the changes of the biological behaviors of the HemECs cells.

Importantly, some studies have reported that AKT and ERK signaling pathways are involved in the pathogenesis of canine haemangiomas ${ }^{16}$ and cerebral cavernous malformation. ${ }^{17}$ Blockade of these pathways may suppress cell proliferation and induce apoptosis 
and cycle arrest in proliferative phase HemECs. ${ }^{18}$ VEGF/NEGFR2 signaling can be indirectly implicated in endothelial cell growth in proliferating infantile HemECs, ${ }^{19}$ and tumor angiogenesis via regulation of AKT and ERK pathways. $^{20}$

Whether VEGFR2 directly regulates AKT and ERK pathways in HemECs need to be further explored.

\section{Materials and Methods}

\section{Materials}

The primary HemECs used in the experiments was from Institute of Biochemistry and Cell Biology (Shanghai, China). The lentivirus vector Lv-shVEGFR2/Lv-VEGFR2, negative control vector and virion-packaging elements were from Genechem (Shanghai, China). The primer of VEGF2R was synthesized by ABI (USA). All antibodies were from Santa Cruz Biotechnology (Dallas, TX, USA).

\section{Drugs and reagents}

Delphinidin (>98\% pure), a VEGFR2 inhibitor was obtained from Extrasynthase (Lyon, France). Dulbecco's Modified Eagle medium (DMEM) and fetal bovine serum (FBS) were from Thermo Fisher Scientific Inc (Waltham, MA, USA); TRIzol Reagent and Lipofectamine 2000 were from Invitrogen (Carlsbad, CA, USA); M-MLV Reverse Transcriptase was from Promega (Madison, WI, USA); SYBR Green Master Mixture was from Takara (Otsu, Japan). ECL-PLUS/Kit was from GE Healthcare (Piscataway, NJ, USA). Cell apoptosis kit [Propidium Iodide (PI), RNase A, Annexin V-FITC] was from KeyGEN biology (Nanjing, China).

\section{Tissue samples}

Fifty-five freshly resected human HA samples were collected from Department of General Surgery Affiliated with Xinhua Hospital, and were classified according to International Society for the Study on Vascular Anomalies (ISSVA) criteria. Tissues and clinical information were summarized in Table 1 and obtained as part of an approved study at Shanghai Jiao Tong University School of Medicine. There were 30 cases of proliferating phase HAs and 25 cases of involuting phase
HAs. A portion of each tissue sample was fixed with $10 \%$ formalin for histopathological and IHC examination. All HA tissues were diagnosed by two independent pathologists.

Table 1. Clinicopathologic characteristics of hemangioma patients.

\begin{tabular}{lc} 
Variables & Number of cases \\
Number of patients & $55(100 \%)$ \\
Age (yrs) & \\
$\leq 1$ & $32(58.2 \%)$ \\
1 & $23(41.8 \%)$ \\
\hline Gender & \\
$\quad$ Male & $25(45.5 \%)$ \\
Female & $30(54.5 \%)$ \\
Location & \\
Head and neck & $34(61.8 \%)$ \\
Trunk and limbs & $21(38.2 \%)$ \\
Treatment & \\
Yes & $0(0 \%)$ \\
No & $55(100 \%)$ \\
Classification & \\
Proliferating phase & $30(54.5 \%)$ \\
Involuting phase & $25(45.5 \%)$ \\
\hline
\end{tabular}
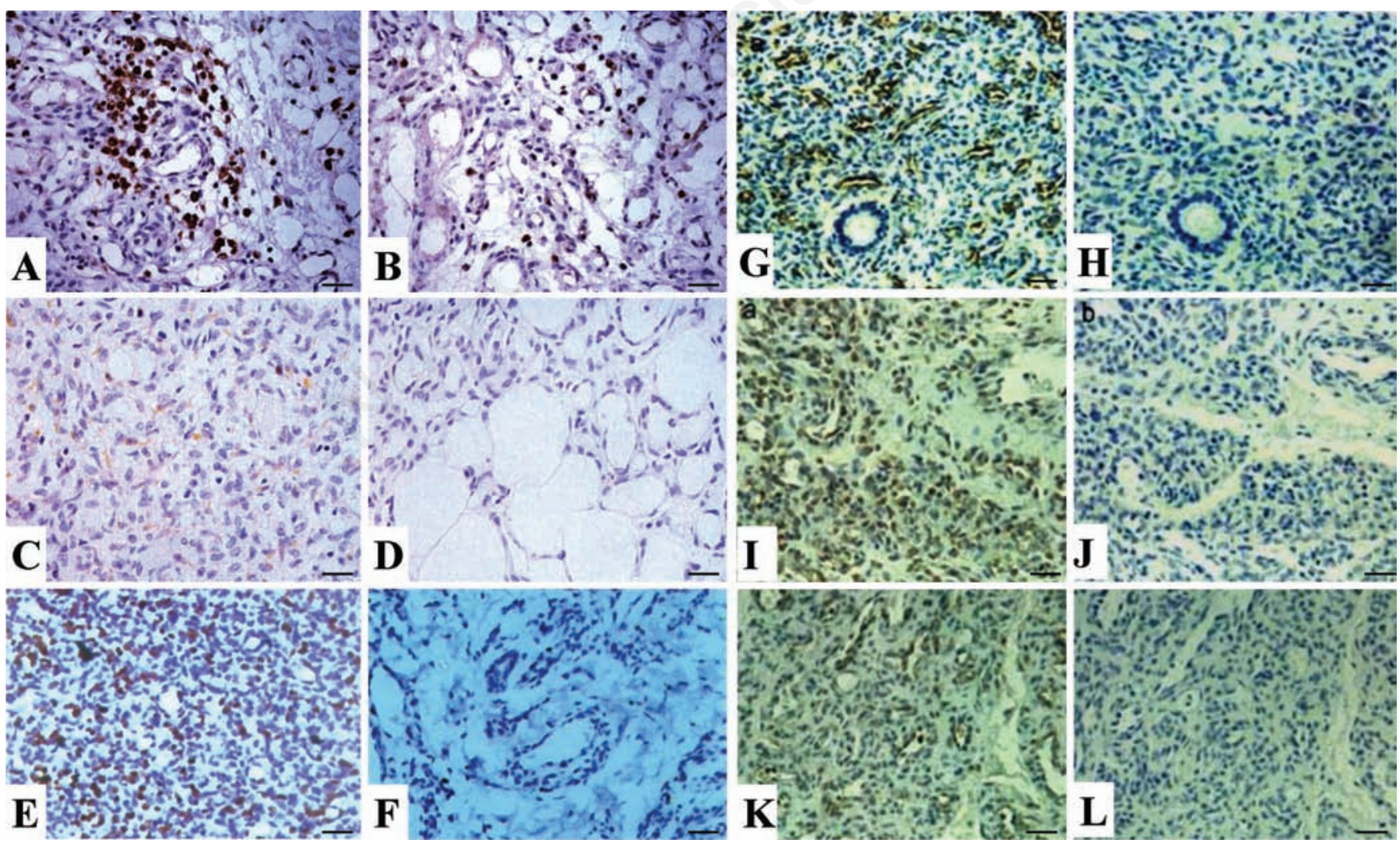

Figure 1. Expression of VEGF, VEGFR2, Ki-67, Glut-1, p-AKT and p-ERK in human HAs ( $\times 400)$. The positive expression of these proteins, mainly localized in the cytoplasm or cell membrane, was strong in proliferating phase HAs, but week in involuting phase HA. A, C, E, G, I, K) Expression of VEGF, VEGFR2, Ki-67, Glut-1, p-AKT and p-ERK, respectively, in proliferating phase HAs. B, D, F, H, J, L) Expression of VEGF, VEGFR2, Ki-67, Glut-1; J), p-AKT and p-ERK, respectively, in involuting phase Has. Scale bars: $37.5 \mu \mathrm{m}$. 


\section{Immunohistochemical staining}

Tissue microarray sections were processed for immunohistochemical (IHC) analysis of VEGF, VEGFR2, Ki-67, Glut-1, p-AKT and p-ERK proteins as follows. Immunohistochemical examinations were carried out on $3 \mathrm{~mm}$ thick sections. For anti-VEGF, VEGFR2, Ki-67, Glut-1, p-AKT and p-ERK immunohistochemistry, unmasking was performed with $10 \mathrm{mM}$ sodium citrate buffer, $\mathrm{pH} 6.0$, at $90^{\circ} \mathrm{C}$ for $30 \mathrm{~min}$. For anti-VEGF, VEGFR2, Ki-67, Glut-1, p-AKT and pERK immunohistochemistry, antigen unmasking was not necessary. Sections were incubated in $0.03 \%$ hydrogen peroxide for $10 \mathrm{~min}$ at room temperature, to remove endogenous peroxidase activity, and then in blocking serum ( $0.04 \%$ bovine serum albumin, A2153, SigmaAldrich, Shanghai, China; and 0.5\% normal goat serum X0907, Dako Corporation, Carpinteria, CA, USA, in PBS) for $30 \mathrm{~min}$ at room temperature. Anti-VEGF and VEGFR2, Ki67, Glut-1, p-AKT and p-ERK antibodies were used at a dilution of 1:200. The antibody was incubated overnight at $4^{\circ} \mathrm{C}$. Sections were then washed three times for $5 \mathrm{~min}$ in PBS. Non-specific staining was blocked with $0.5 \%$ casein and 5\% normal serum for $30 \mathrm{~min}$ at room temperature. Finally, staining was developed using diaminobenzidine substrate, and sections were counterstained with hematoxylin. Normal serum or PBS was used to replace anti-VEGF, VEGFR2, Ki-67, Glut-1, pAKT and p-ERK antibodies in negative controls. The expression of VEGF, VEGFR2, Ki-67, Glut-1, p-AKT and p-ERK was semiquantitatively estimated as the total IHC staining score, which was calculated as the sum of a proportion score. The score reflected the fraction of positive staining cells $(-,<5 \% ;+, 5 \%$ $25 \%$; ++, >25\% 50\%; +++, >50\%).

\section{TUNEL assay}

Apoptosis was detected by the TdT-mediated dUTP nick end labeling (TUNEL) method for HA cells $\left(1 \times 10^{5}\right.$ clones $)$. Briefly, sections were dewaxed, incubated with blocking solution $\left(0.3 \% \mathrm{H}_{2} \mathrm{O}_{2}\right.$ in double distilled water) for 30 min, and permeabilized with $0.1 \%$ Triton X-100 in PBS for two min on ice. Apoptosis was detected using an in-situ cell death kit (Boehringer Mannheim, Germany). Positive cells were visualized by fluorescence microscopy. As a control, the reaction mixture was incubated without enzyme to detect nonspecific staining. The apoptotic index was calculated from the ratio of the number of positively stained tumor cells to the total number of tumor cells counted per section.

\section{Cell culture and transfection}

The proliferating phase HemECs $\left(1 \times 10^{5}\right.$ clones) was cultured in DMEM medium supplemented with 10\% heat-inactivated FBS, 100
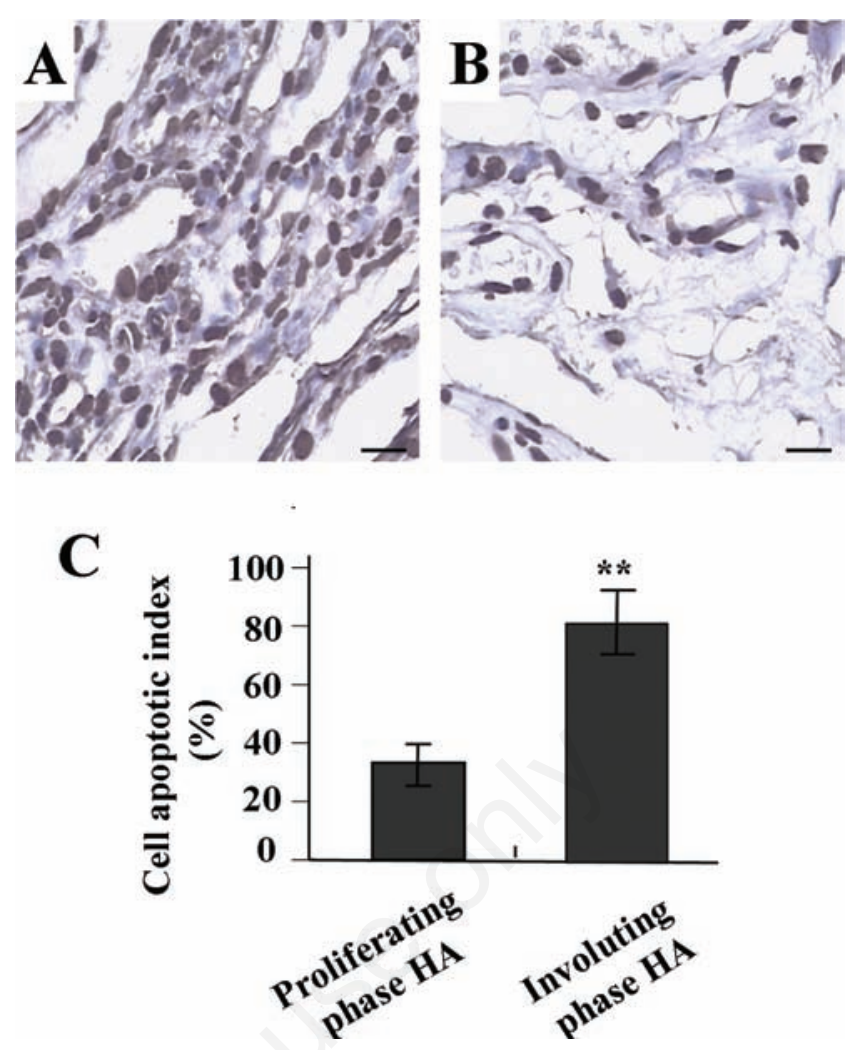

Figure 2. Apoptotic indexes of human HAs examined by TUNEL assay ( $\times 400)$. A) Positive staining of apoptotic cells in involuting phase Has. B) Positive staining of apoptotic cells in proliferating phase Has. C) The apoptotic cells were dramatically decreased in proliferating phase $\mathrm{HAs}$, but increased in involuting phase HAs $(\mathrm{P}<0.01)$. Scale bars: $37.5 \mu \mathrm{m}$.
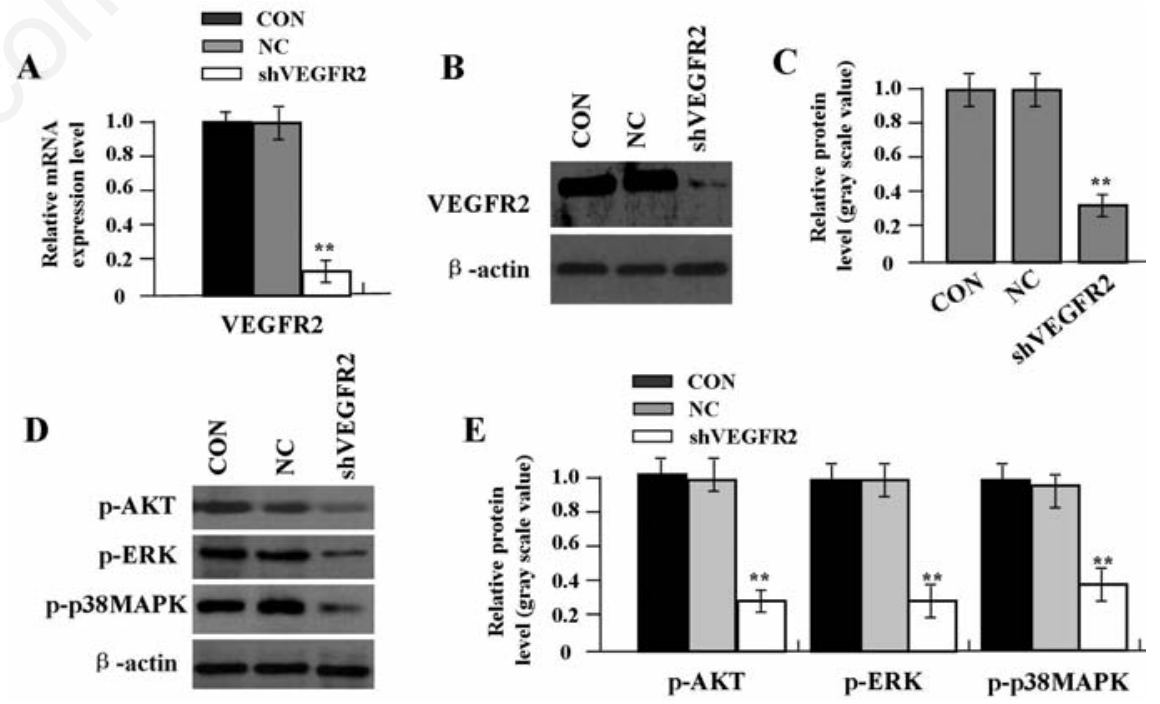

Figure 3. Effect of VEGFR2 on p-AKT. p-ERK and p-p38MAPK expression in primary HemECs. A) Expression level of VEGFR2 mRNA was reduced in shVEGFR2 group compared with CON and NC groups $(* * P<0.01)$. B,C) Expression of VEGFR2 protein was markedly downregulated in shVEGFR2 group compared with CON and NC groups $\left.\left({ }^{* *} \mathrm{P}<0.01\right) . \mathrm{D}, \mathrm{E}\right)$ Expression of p-AKT, p-ERK and p-p38MAPK was also downregulated in shVEGFR2 group compared with CON and NC groups $(* * P<0.01)$. 
$\mathrm{U} / \mathrm{mL}$ of penicillin and $100 \mu \mathrm{g} / \mathrm{mL}$ of streptomycin. They were all placed in a humidified atmosphere containing $5 \% \mathrm{CO}_{2}$ at $37^{\circ}$. LvshVEGFR2/Lv-VEGFR2 and negative control virus were transfected into proliferating phase HemECs. Cells were subcultured at a 1:5 dilution in medium containing $300 \mu \mathrm{g} / \mathrm{mL}$ G418 (an aminoglycoside antibody, commonly used stable transfection reagent in molecular genetic testing). On the day of transduction, HemECs were replated at $5 \times 10^{4}$ cells/well in 24 -well plates containing serum-free growth medium with polybrene $(5 \mathrm{mg} / \mathrm{mL})$. When reached $50 \%$ confluence, cells were transfected with recombinant experimental virus or control virus at the optimal MOI (multiplicity of infection) of 50 , and cultured at $37^{\circ} \mathrm{C}$ and $5 \% \mathrm{CO}_{2}$ for $4 \mathrm{~h}$. Then supernatant was discarded and serum containing growth medium was added. At 4 days of post-transduction, transduction efficiency was measured by the frequency of green fluorescent protein (GFP)positive cells. The clone in which Lv-shVEGFR2 vectors transfected was named as shVEGFR2 group, the negative control vectors transfected was named as negative control (NC) group. The untreated HemECs was named as control (CON) group.

\section{Quantitative real-time PCR}

To quantitatively determine the mRNA expression level of VEGFR2 in proliferating phase HemECs $\left(1 \times 10^{5}\right.$ clones), Real-time PCR was used. Total RNA of each clone was extracted with TRIzol according to the manufacturer's protocol. Reverse-transcription was carried out using M-MLV and cDNA amplification was carried out using SYBR Green Master Mix kit according to the manufacturer's protocol. VEGFR2 gene was amplified using specific oligonucleotide primer and human glyceraldehyde-3-phosphate dehydrogenase (GAPDH) gene was used as an endogenous control. The PCR primer sequences of VEGFR2 gene were as follows:5'-CTTCGAAGCATCAGCATAAGAAACT-3' and 5'-TGGTCATCAGCCC ACTGGAT-3'; GAPDH, 5'-AACGACCCCTTCATTGAC-3' and 5'-TCCACGAC ATACTCAGCAC-3'. Data were analyzed using the comparative $\mathrm{Ct}$ method $\left(2^{-\Delta \Delta c t}\right)$. Three separate experiments were performed for each clone.

\section{Western blot assay}

The proliferating phase HemECs $\left(1 \times 10^{5}\right.$ clones) was harvested and extracted using lysis buffer (Tris-HCl, SDS, Mercaptoethanol, Glycerol). Cell extracts were boiled for $5 \mathrm{~min}$ in loading buffer and then equal amount of cell extracts were separated on 15\% SDS-PAGE gels. Separated protein bands were transferred into polyvinylidene fluoride (PVDF) membranes and the membranes were blocked in $5 \%$ skim milk powder. The primary antibodies against
VEGFR2, p-AKT, p-ERK, p-p38MAPK, Ki-67 and CAS-3 were diluted according to the instructions of antibodies and incubated overnight at $4^{\circ} \mathrm{C}$. Then, horseradish peroxidase-linked secondary antibodies were added at a dilution ratio of 1:1000, and incubated at room temperature for $2 \mathrm{~h}$. The membranes were washed with PBS for three times and the immunoreactive bands were visualized using ECL-PLUS/Kit according to the kit's instruction. The relative protein level in HemECs was normalized to $\beta$-actin concentration. Three separate experiments were performed for each clone.

\section{Cell viability assay}

Cell viability was analyzed with the MTT assay. Briefly, HemECs infected with LvshVEGFR2 or treated with Delphinidin (0, 10 $\mu \mathrm{M}, 50 \mu \mathrm{M})$ were incubated in 96-well-plates at a density of $1 \times 10^{5}$ cells per well with DEME medium supplemented with $10 \%$ FBS. Cells were treated with $20 \mu \mathrm{L}$ MTT dye at $0,24 \mathrm{~h}, 48$ $\mathrm{h}$ and $72 \mathrm{~h}$, and then incubated with $150 \mu \mathrm{L}$ of DMSO for $5 \mathrm{~min}$. The color reaction was measured at $570 \mathrm{~nm}$ with enzyme immunoassay analyzer (Bio-Rad, American). The proliferation activity was calculated for each clone.

\section{Cell apoptosis analysis}

To detect cell apoptosis, the proliferating phase HemECs $\left(1 \times 10^{5}\right.$ clones $)$ was trypsinized, washed with cold PBS and resuspended in binding buffer according to the instruction of the apoptosis kit. FITCAnnexinV and PI were added to the fixed cells for $20 \mathrm{~min}$ in darkness at room temperature. Then, Annexin V binding buffer was added to the mixture before the fluorescence was measured on FACsort flow cytometer. The cell apoptosis was analyzed using the Cell Quest software (Becton Dickinson, Franklin Lakes, NJ, USA). Three separate experiments were performed for each clone.
A

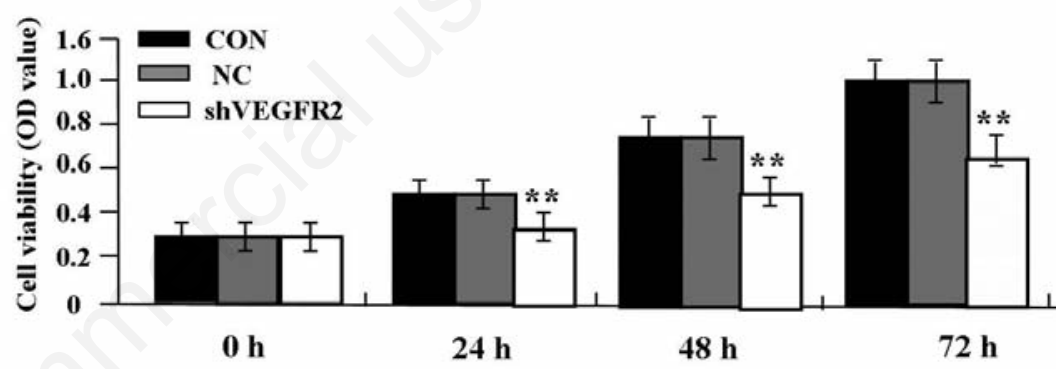

B

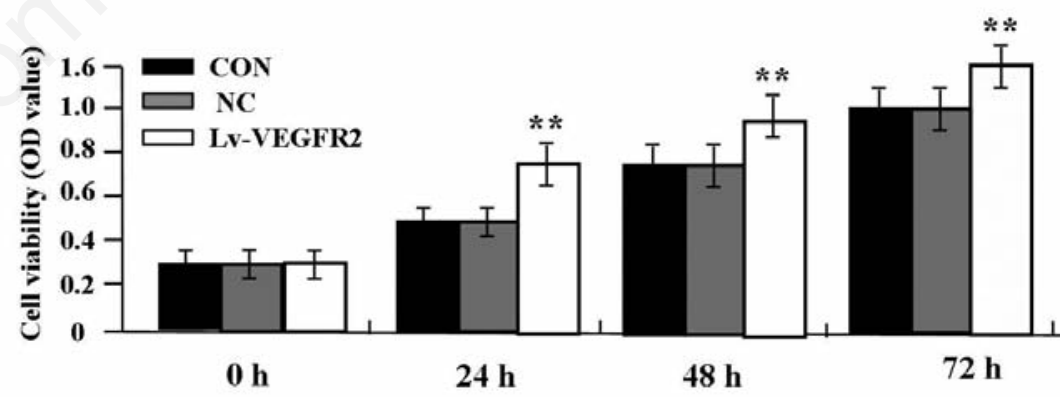

C

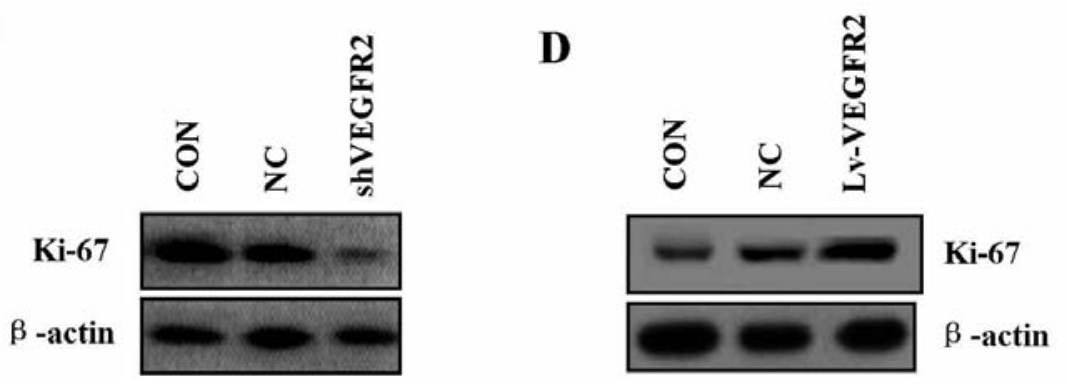

Figure 4. Effect of VEGFR2 on cell viability. A) Knockdown of VEGFR2 significantly diminished cell viability. B) Overexpression of VEGFR2 promoted proliferation in a timedependent manner in HemECs $\left({ }^{* *} \mathrm{P}<0.01\right)$. C) Protein expression of $\mathrm{Ki}-67$ was significantly decreased in shVEGFR2 group. D) Expression of Ki-67 was increased in LvVEGFR2 group compared with CON and NC groups $(* * \mathrm{P}<0.01)$. 


\section{Statistical analysis}

SPSS 20.0 was used for the statistical analysis. One-way analysis of variance (ANOVA) was used to analyze the differences between groups. The Fisher's Least Significant Difference (LSD) method of multiple comparisons was used when the probability for ANOVA was statistically significant. Statistical significance was $\mathrm{P}<0.05$.

\section{Results}

Expression of VEGF, VEGFR2, Ki67, Glut-1, p-AKT and p-ERK in human hemangiomas

The expression of VEGF, VEGFR2, Ki-67, Glut-1, p-AKT and p-ERK proteins was evaluated using IHC analysis. As shown in Figure $1 \mathrm{~A}-$ $\mathrm{L}$, the positive staining of VEGF, VEGFR2, Ki67, Glut-1, p-AKT and p-ERK proteins was found strong, and was mainly localized in the cytoplasm or cell membrane in proliferating phase HA, but that of these proteins was week in involuting phase HA. As indicated in Table 2, the positive rates of VEGF, VEGFR2, Ki-67, Glut1, p-AKT and p-ERK were significantly higher in proliferating phase HA than those in involuting phase $\mathrm{HA}(\mathrm{P}<0.01)$.

\section{Apoptotic index of human hemangioma}

As shown in Figure 2, using TUNEL method we found that the apoptotic indexes were dramatically decreased in proliferating phase HA, but increased in involuting phase HA. There was a significant difference between these two groups $(\mathrm{P}<0.01)$.

\section{Effect of VEGFR2 knockdown on $\mathrm{p}-\mathrm{AKT}$ and $\mathrm{p}-\mathrm{ERK}$ expression}

To examine whether the expression of VEGFR2 was knocked down by Lv-shVEGFR2 in proliferative phase HemECs, the mRNA expression level of VEGFR2 was measured by Real-time PCR (Figure 3A) An obvious inhibition of VEGFR2 mRNA expression was observed in shVEGFR2 group compared with the $\mathrm{NC}$ and CON groups $\left({ }^{* *} \mathrm{P}<0.01\right)$. As for the protein expression indicated by Western blot assay (Figure 3 B-E), VEGFR2, p-AKT, p-ERK and p-p38MAPK were markedly downregulated in shVEGFR2 group in comparison with the NC and CON groups in primary HemECs $(* * \mathrm{P}<0.01)$.

\section{Effect of VEGFR2 on cell proliferation}

By comparing the viability assessed by MTT assay among different culture days, the cell proliferative activity was evaluated in primary HemECs, and Ki-67 was used to assess cell proliferation. As a result, knockdown of VEGFR2 significantly diminished proliferative activities of HemECs (Figure 4A) while overexpres-
A Delphinidin ( $\mu$ M)

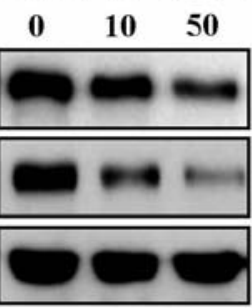

VEGF

VEGFR2

$\beta$-actin

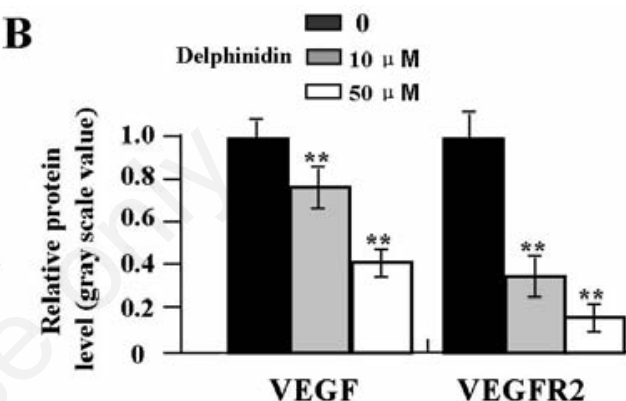

C

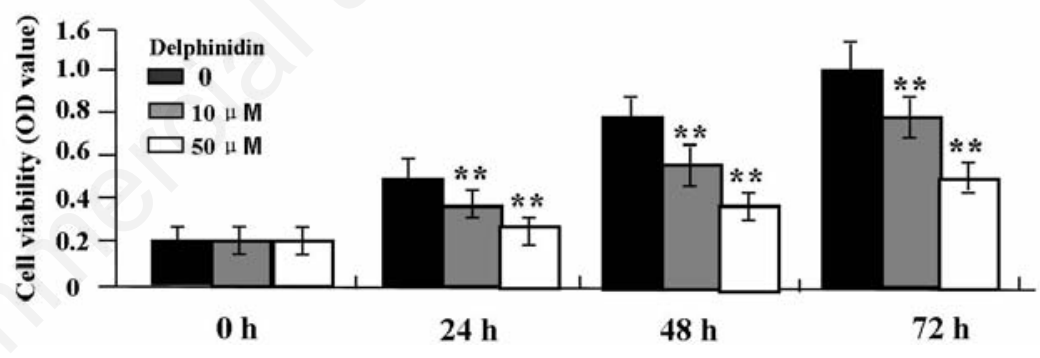

Figure 5. Effect of Delphinidin on cell proliferation. A, B) Delphinidin suppressed the protein expression levels of VEGF and VEGFR2 and cell viability $(C)$ in a dose-dependent manner in primary HemECs $(* * \mathrm{P}<0.01)$.

Table 2. Expression of VEGF, VEGFR2, Ki-67, Glut-1, p-AKT and p-ERK in human hemangiomas.

\begin{tabular}{|c|c|c|c|c|c|c|c|c|c|}
\hline \multirow[t]{2}{*}{ Target } & \multirow[t]{2}{*}{ Group } & \multirow[t]{2}{*}{ Cases } & \multicolumn{4}{|c|}{ n } & \multirow[t]{2}{*}{ Positive rate (\%) } & \multirow[t]{2}{*}{$x^{2}$} & \multirow[t]{2}{*}{$\mathbf{P}$} \\
\hline & & & - & + & ++ & +++ & & & \\
\hline \multirow[t]{2}{*}{ VEGF } & Proliferating phase HAs & 30 & 6 & 7 & 12 & 5 & 80.0 & \multirow[t]{2}{*}{11.712} & \multirow[t]{2}{*}{0.001} \\
\hline & Involuting phase HAs & 25 & 13 & 9 & 3 & 0 & 48.0 & & \\
\hline \multirow[t]{2}{*}{ VEGFR2 } & Proliferating phase HAs & 30 & 8 & 6 & 10 & 6 & 73.3 & \multirow[t]{2}{*}{8.628} & \multirow[t]{2}{*}{0.003} \\
\hline & Involuting phase HAs & 25 & 15 & 6 & 3 & 1 & 40.0 & & \\
\hline \multirow[t]{2}{*}{ Ki-67 } & Proliferating phase HAs & 30 & 7 & 12 & 6 & 5 & 76.7 & \multirow[t]{2}{*}{5.559} & \multirow[t]{2}{*}{0.018} \\
\hline & Involuting phase HAs & 25 & 14 & 6 & 4 & 1 & 44.0 & & \\
\hline \multirow[t]{2}{*}{ Glut-1 } & Proliferating phase HAs & 30 & 5 & 8 & 8 & 9 & 83.3 & \multirow{2}{*}{19.921} & \multirow[t]{2}{*}{$<0.001$} \\
\hline & Involuting phase HAs & 25 & 18 & 5 & 2 & 0 & 28.0 & & \\
\hline \multirow[t]{2}{*}{ p-AKT } & Proliferating phase HAs & 30 & 8 & 13 & 7 & 2 & 73.3 & \multirow[t]{2}{*}{8.512} & \multirow[t]{2}{*}{0.004} \\
\hline & Involuting phase HAs & 25 & 16 & 7 & 2 & 0 & 36.0 & & \\
\hline \multirow[t]{2}{*}{ p-ERK } & Proliferating phase HAs & 30 & 10 & 13 & 5 & 2 & 66.7 & \multirow[t]{2}{*}{8.913} & \multirow[t]{2}{*}{0.003} \\
\hline & Involuting phase HAs & 25 & 18 & 6 & 1 & 0 & 28.0 & & \\
\hline
\end{tabular}

Has, hemangiomas 
sion of VEGFR2 enhanced cell viability (Figure $4 \mathrm{~B})$ in a time-dependent manner compared with $\mathrm{NC}$ and CON groups. In addition, the expression of Ki-67 protein was examined by Western blot. The amount of Ki-67 protein was significantly decreased in shVEGFR2 group (Figure 4C), but increased in Lv-VEGFR2 group (Figure 4D) compared with $\mathrm{NC}$ and $\mathrm{CON}$ groups $(* * \mathrm{P}<0.01)$.

\section{Effect of Delphinidin, a VEGFR2 inhibitor on cell viability}

The primary HemECs were pretreated with different concentrations of Delphinidin $(0,10$ $\mu \mathrm{M}, 50 \mu \mathrm{M})$, and the expression of VEGF and VEGFR2 was examined by Western blot and cell viability was assessed by MTT assay. It was found that Delphinidin could markedly downregulate the expression of VEGF and VEGFR2 (Figure $5 \mathrm{~A}, \mathrm{~B}$ ) and reduce cell viability (Figure $5 \mathrm{C}$ ) in a dose-dependent manner in primary HemECs.

\section{Effect of VEGFR2 on cell apoptosis}

To determine whether VEGFR2 influenced cell apoptosis in primary HemECs, flow cytometric analysis was performed. Cell apoptotic indexes were significantly increased in shVEGFR2 group (Figure 6 A,B), but was decreased in Lv-VEGFR2 group (Figure 6 C,D) compared with $\mathrm{NC}$ and $\mathrm{CON}$ groups $\left({ }^{* *} \mathrm{P}<0.01\right)$. In order to determine whether VEGFR2 regulated the expression of CAS-3 through translational level, the expression of CAS-3 protein was examined by Western blot. The amount of CAS-3 protein was significantly increased in shVEGFR2 group (Figure 6E), but was decreased in Lv-VEGFR2 group (Figure 6F) compared with $\mathrm{NC}$ and CON groups $\left({ }^{* *} \mathrm{P}<0.01\right)$.

\section{Discussion}

VEGF and its receptor VEGFR have been shown to play major roles not only in physiological but also in pathological angiogenesis including HA. The VEGF-VEGFR system is an important target for anti-angiogenic therapy in cancer. ${ }^{21}$ Overexpression of VEGF/NEGFR-2 signaling plays an important role in promoting invasion and migration of pancreatic cancer cells, ${ }^{22}$ and is associated with the prognosis in patients with lung squamous cell carcinoma. ${ }^{23}$ Moreover, VEGFR-2-mediated signal contributes to proliferation of vascular tumor cells, ${ }^{24}$ while blockade of this signaling through VEGF inhibitor bevacizumab provides a potential treatment of for hepatic HA. ${ }^{25}$

However, soluble VEGFR2 in some studies have been found to be lowly expressed in invasive colorectal cancer, ${ }^{26}$ while PVITR02-hm-
sVEGFR2, which may be a novel DNA vaccine for the anti-tumor therapy, can inhibit angiogenesis. ${ }^{27}$ To understand the role of VEGFR2 signaling in HAs, we examined the expression of VEGF and VEGFR2 in human HA tissue using IHC, and found that, the expression of VEGF, VEGFR2, Ki-67, Glut-1, p-AKT and p-ERK was significantly increased in proliferating phase HAs, but decreased in involuting phase HAs, suggesting VEGFR2 signaling may be implicated in the development of HAs. Furthermore, blockade of the VEGF/VEGFR pathway may be a rational and effective therapy for certain tumor patients including Has. ${ }^{28}$ The agents, which can inhibit this signaling transduction, need be identified and confirmed to be beneficial to the patients. Elpamotide is an immunogenic peptide derived from VEGFR2, and inhibits tumor angiogenesis in advanced solid tumors, indicating a potentially valuable approach to cancer. ${ }^{29}$ Targeted silencing of VEGFR2 and EGFR expression by siRNA, combined with cisplatin is found to effectively inhibit tumor growth and extend the survival time of NSCLC xenografts. ${ }^{30}$ Intriguingly, propranolol markedly inhibits HA and normal endothelial cell function, including cell proliferation, migration, and formation of the cytoskeleton coincident through inhibition of VEGFR2 and p38 signaling. ${ }^{31}$ In the present study, our findings showed that, knockdown of VEGFR2 significantly suppressed cell proliferation and induced cell apoptosis, but overexpression of VEGFR2 promoted proliferation and blocked apoptosis in primary HemECs. Importantly, Delphinidin, a VEGFR2 inhibitor could markedly downregulated the expression of VEGF and VEGFR2, and reduce cell viability. Some studies also support our findings, indicating that up-regulation of the autocrine VEGF/NEGFR2 loop induces general resistance to apoptotic stimuli in HemECs. ${ }^{32}$ These reports suggest that, VEGFR2 signaling might promote the progression of HAs, and represent a novel therapeutic target.

There is growing evidence that activation of AKT and ERK pathways plays a significant role in cancer, and offers targeted therapy approaches for cancer. ${ }^{33}$ Bevacizumab inhibits tumor growth and attenuates VEGFR2-induced p-AKT and p-ERK, ${ }^{34}$ and usnic acid ${ }^{35}$ or luteolin ${ }^{36}$ also suppresses tumor angiogenesis and growth by suppressing VEGFR2-mediated ERK and AKT signaling pathways. More importantly, inhibition of HA development in a syngeneic mouse model correlates with the inhibition of AKT and ERK activity. ${ }^{37}$ In the present study, we found that, knockdown of VEGFR2 could

\section{A}
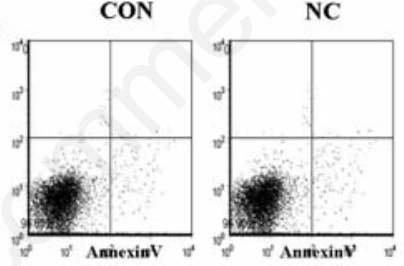

C

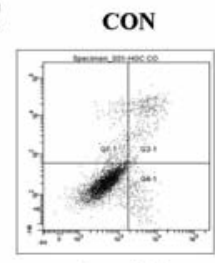

Annexinv

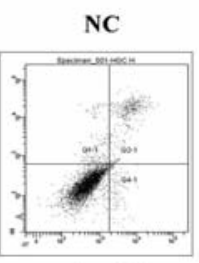

AnnexinV
ShVEGFR2

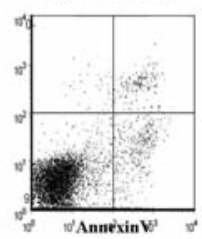

Lv-VEGFR2

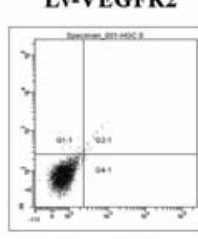

AnnexinV
B

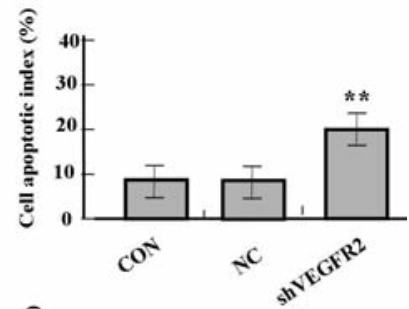

D

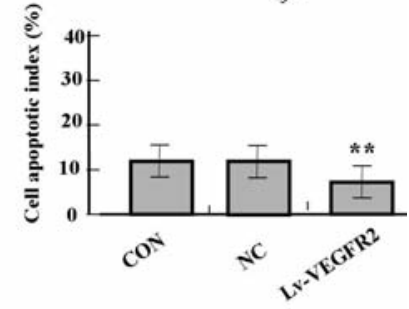

E

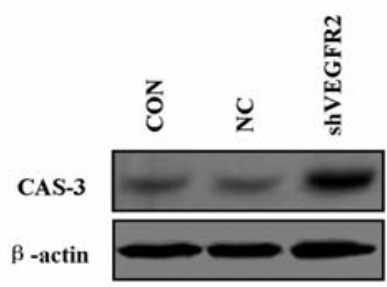

F

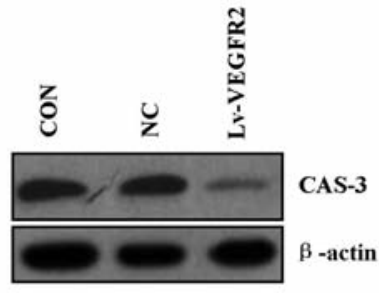

Figure 6. Effect of VEGFR2 on cell apoptosis. A, B) Flow cytometric analysis showed that cell apoptotic indexes of HemECs were increased in shVEGFR2 group, but were decreased in Lv-VEGFR2 group $(C, D)$ compared with CON and NC groups $\left({ }^{* *} P<0.01\right)$. E) The protein expression of CAS-3 was upregulated in shVEGFR2 group, but downregulated in Lv-VEGFR2 group $(\mathrm{F})$ compared with CON and $\mathrm{NC}$ groups $(* * \mathrm{P}<0.01)$. 
significantly downregulate the expression of pAKT, p-ERK, and p-p38MAPK in primary HemECs, suggesting that VEGFR2 signaling may be implicated in HA proliferation and inhibition of apoptosis via regulation of the AKT, ERK and MAPK signaling pathways.

Many studies have proved that Ki- 67 can be used as the biomarker of the tumor in response to cellular proliferation. It also serves as a diagnostic tool to distinguish between benign and malignant vascular lesions including Has. ${ }^{38}$ In addition, CAS-3 is also involved in the development of HAs, and promotes the switch of HAs from proliferation to involution by inducing the apoptosis of HAs endothelia. ${ }^{39}$ AKT, ERK and MAPK signaling pathways have been found to be correlated with the expression of Ki-67 in human cancers. ${ }^{40}$ In the present study, we also found that, knockdown of VEGFR2 could significantly downregulate the expression of $\mathrm{Ki}-67$, and upregulate the expression of CAS-3 in primary HemECs, suggesting that AKT, ERK and MAPK pathways might mediate the effects of VEGFR2 on HAs through regulation of expression of $\mathrm{Ki}-67$ and CAS-3.

In conclusion, our findings indicate that the expression of VEGF and VEGFR2 is significantly elevated in proliferating phase HAs, but decreased in involuting phase HAs. Knockdown of VEGFR2 inhibits proliferation and induces cell apoptosis, but overexpression of VEGFR2 promotes proliferation and blocks apoptosis in primary HemECs possibly through regulation of the AKT and ERK pathways, suggesting that VEGFR2 may be a potential therapeutic target for the treatment of human hemangioma.

\section{References}

1. Mulliken JB, Fishman SJ, Burrows PE. Vascular anomalies. Curr Probl Surg 2000;37:517-84.

2. Mabeta P, Pepper MS. Hemangiomas-current therapeutic strategies. Int J Dev Biol 2011;55:431-7.

3. Shibuya M. Vascular endothelial growth factor and its receptor system: physiological functions in angiogenesis and pathological roles in various diseases. $\mathrm{J}$ Biochem 2013;153:13-9.

4. Dhakal HP, Naume B, Synnestvedt M, Borgen E, Kaaresen R, Schlichting E, et al. Expression of vascular endothelial growth factor and vascular endothelial growth factor receptors 1 and 2 in invasive breast carcinoma: prognostic significance and relationship with markers for aggressiveness. Histopathology 2012;61:350-64.

5. Uzunoglu FG, Kaufmann C, Wikman H,
Güngör C, Bohn BA, Nentwich MF, et al. Vascular endothelial growth factor receptor 2 gene polymorphisms as predictors for tumor recurrence and overall survival in non-small-cell lung cancer. Ann Surg Oncol 2012;19:2159-68.

6. Woollard DJ, Opeskin K, Coso S, Wu D, Baldwin ME, Williams ED. Differential expression of VEGF ligands and receptors in prostate cancer. Prostate 2013;73:563-72.

7. Tabata S, Goi T, Nakazawa T, Kimura Y, Katayama K, Yamaguchi A. Endocrine gland-derived vascular endothelial growth factor strengthens cell invasion ability via prokineticin receptor 2 in colon cancer cell lines. Oncol Rep 2013;29:459-63.

8. Paesler J, Gehrke I, Poll-Wolbeck SJ, Kreuzer KA. Targeting the vascular endothelial growth factor in hematologic malignancies. Eur J Haematol 2012;89: 373-84.

9. Medici D, Olsen BR. Rapamycin inhibits proliferation of hemangioma endothelial cells by reducing HIF-1-dependent expression of VEGF. PLoS One 2012;7:e42913.

10. Walter JW, North PE, Waner M, Mizeracki A, Blei F, Walker JW, et al. Somatic mutation of vascular endothelial growth factor receptors in juvenile hemangioma. Genes Chromosomes Cancer 2002; 33:295-303.

11. Kleinman ME, Tepper OM, Capla JM, Bhatt KA, Ceradini DJ, Galiano RD, et al. Increased circulating AC133+ CD34+ endothelial progenitor cells in children with hemangioma. Lymphat Res Biol 2003; 1:301-7.

12. Nicolae C, Olsen BR. Unexpected matrix diseases and novel therapeutic strategies. Cell Tissue Res 2010; 339:155-65.

13. Li Y, Xia Y, Jin B. Effect of anti-KDR antibody on the proliferation of hemangioma vascular endothelial cells in vitro. $\mathrm{J}$ Huazhong Univ Sci Technolog Med Sci 2007;27:551-3.

14. Przewratil P, Sitkiewicz A, Andrzejewska E. Soluble receptors for vascular endothelial growth factor (sVEGFR1/sVEGFR2) in infantile hemangioma. Growth Factors 2010;28:417-25.

15. Lu KV, Chang JP, Parachoniak CA, Pandika MM, Aghi MK, Meyronet D, et al. VEGF inhibits tumor cell invasion and mesenchymal transition through a MET/VEGFR2 complex. Cancer Cell 2012;22:21-35.

16. Murai A, Abou Asa S, Kodama A, Sakai H, Hirata A, Yanai T. mmunohistochemical analysis of the AKT/mTOR/4E-BP1 signalling pathway in canine haemangiomas and haemangiosarcomas. J Comp Pathol 2012;147:430-40.

17. Ma X, Zhao H, Shan J, Long F, Chen Y, Chen Y, et al. PDCD10 interacts with
Ste20-related kinase MST4 to promote cell growth and transformation via modulation of the ERK pathway. Mol Biol Cell 2007;18:1965-78.

18. Ou JM, Qui MK, Dai YX, Dong Q, Shen J, Dong $\mathrm{P}$, et al. Combined blockade of AKT/mTOR pathway inhibits growth of human hemangioma via downregulation of proliferating cell nuclear antigen. Int $\mathrm{J}$ Immunopathol Pharmacol 2012;25:945-53.

19. Lee J Jr, Chen CH, Chen YH, Huang MJ, Huang J, Hung JS, et al. COSMC is overexpressed in proliferating infantile hemangioma and enhances endothelial cell growth via VEGFR2. PLoS One 2013;8: e56211.

20. Zhang L, Ji Q, Liu X, Chen X, Chen Z, Qiu $\mathrm{Y}$, et al. Norcantharidin inhibits tumor angiogenesis via blocking VEGFR2/ MEK/ERK signaling pathways. Cancer Sci 2013;104:604-10.

21. Shibuya M. Vascular Endothelial Growth Factor (VEGF) and Its Receptor (VEGFR) Signaling in Angiogenesis: A Crucial Target for Anti- and Pro-Angiogenic Therapies. Genes Cancer 2011;2:1097-105.

22. Doi Y, Yashiro M, Yamada N, Amano R, Noda S, Hirakawa K. VEGF-A/VEGFR-2 signaling plays an important role for the motility of pancreas cancer cells. Ann Surg Oncol 2012;19:2733-43.

23. Pajares MJ, Agorreta J, Larrayoz M, Vesin A, Ezponda T, Zudaire I, et al. Expression of tumor-derived vascular endothelial growth factor and its receptors is associated with outcome in early squamous cell carcinoma of the lung. J Clin Oncol 2012; 30:1129-36.

24. Tokuyama W, Mikami T, Masuzawa M, Okayasu I. Autocrine and paracrine roles of VEGF/VEGFR-2 and VEGF-C/VEGFR-3 signaling in angiosarcomas of the scalp and face. Hum Pathol 2010;41:407-14.

25. Mahajan D, Miller C, Hirose K, McCullough A, Yerian L. Incidental reduction in the size of liver hemangioma following use of VEGF inhibitor bevacizumab. J Hepatol 2008;49:867-70.

26. Shantha Kumara HM, Cabot JC, Hoffman A, Luchtefeld M, Kalady MF, Hyman N, et al. Minimally invasive colon resection is associated with a transient increase in plasma sVEGFR1 levels and a decrease in sVEGFR2 levels during the early postoperative period. Surg Endosc 2009;23:694-9.

27. Jin L, Zhao YW, Li ZM, Zhao CJ, Wang XF, Zhang L, et al. [Anti-tumor effects induced by the DNA vaccine coding human and mouse soluble VEGFR2].[Article in Chinese]. Sichuan Da Xue Xue Bao Yi Xue Ban 2010;41:563-6.

28. Park MS, Ravi V, Araujo DM. Inhibiting the VEGF-VEGFR pathway in angiosarcoma, 
epithelioid hemangioendothelioma, and hemangiopericytoma/solitary fibrous tumor. Curr Opin Oncol 2010;22:351-5.

29. Okamoto I, Arao T, Miyazaki M, Satoh T, Okamoto K, Tsunoda T, et al. Clinical phase I study of elpamotide, a peptide vaccine for vascular endothelial growth factor receptor 2 , in patients with advanced solid tumors. Cancer Sci 2012;103:2135-8.

30. Chen S, Liu X, Gong W, Yang H, Luo D, Zuo $\mathrm{X}$, et al. Combination therapy with VEGFR2 and EGFR siRNA enhances the antitumor effect of cisplatin in non-small cell lung cancer xenografts. Oncol Rep 2013;29:260-8.

31. Stiles J, Amaya C, Pham R, Rowntree RK, Lacaze M, Mulne A, et al. Propranolol treatment of infantile hemangioma endothelial cells: A molecular analysis. Exp Ther Med 2012;4:594-604.

32. Ji Y, Chen S, Li K, Xiao X, Xu T, Zheng S. Up-regulated autocrine VEGF/VEGFR-2 loop prevents apoptosis in hemangioma- derived endothelial cells. Br J Dermatol 2014;170:78-86.

33. Davies MA. The role of the PI3K-AKT pathway in melanoma. Cancer J 2012;18:142-7.

34. Shin SJ, Hwang JW, Ahn JB, Rha SY, Roh JK, Chung HC. Circulating vascular endothelial growth factor receptor 2/pAktpositive cells as a functional pharmacodynamic marker in metastatic colorectal cancers treated with antiangiogenic agent. Invest New Drugs 2013;31:1-13.

35. Song Y, Dai F, Zhai D, Dong Y, Zhang J, Lu B, et al. Usnic acid inhibits breast tumor angiogenesis and growth by suppressing VEGFR2mediated AKT and ERK1/2 signaling pathways. Angiogenesis 2012;15:421-32.

36. Pratheeshkumar P, Son YO, Budhraja A, Wang X, Ding S, Wang L, et al. Luteolin inhibits human prostate tumor growth by suppressing vascular endothelial growth factor receptor 2-mediated angiogenesis. PLoS One 2012;7:e52279.
37. Mabeta P, Pepper MS. Inhibition of hemangioma development in a syngeneic mouse model correlates with bcl-2 suppression and the inhibition of Akt kinase activity. Angiogenesis 2012;15:131-9.

38. Shin SJ, Lesser M, Rosen PP. Hemangiomas and angiosarcomas of the breast: diagnostic utility of cell cycle markers with emphasis on Ki-67. Arch Pathol Lab Med 2007;131:538-44.

39. Yang H, Deng C, Shen S, Zhang D, Yu Y. Expression and significance of Bcl-2, Bax, Fas and caspase-3 in different phases of human hemangioma. J Huazhong Univ Sci Technolog Med Sci 2006;26:402-4.

40. Ghosh PM, Malik SN, Bedolla RG, Wang Y, Mikhailova M, Prihoda TJ, et al. Signal transduction pathways in androgendependent and -independent prostate cancer cell proliferation. Endocr Relat Cancer 2005;12:119-34. 